\title{
Product News
}

\section{Crystal-Clear Images Reveal Secrets of Energy- Efficient Catalysts and Novel Materials}

To develop new materials and improve the efficiency of the chemical transformations that will someday provide renewable energy for our homes and businesses, scientists and engineers at Pacific Northwest National Laboratory, Richland, Washington, worked with building architects to design a space within the new Energy Sciences Center that is protected from magnetic fields, vibrations, and temperature fluctuations that can cause distorted images.

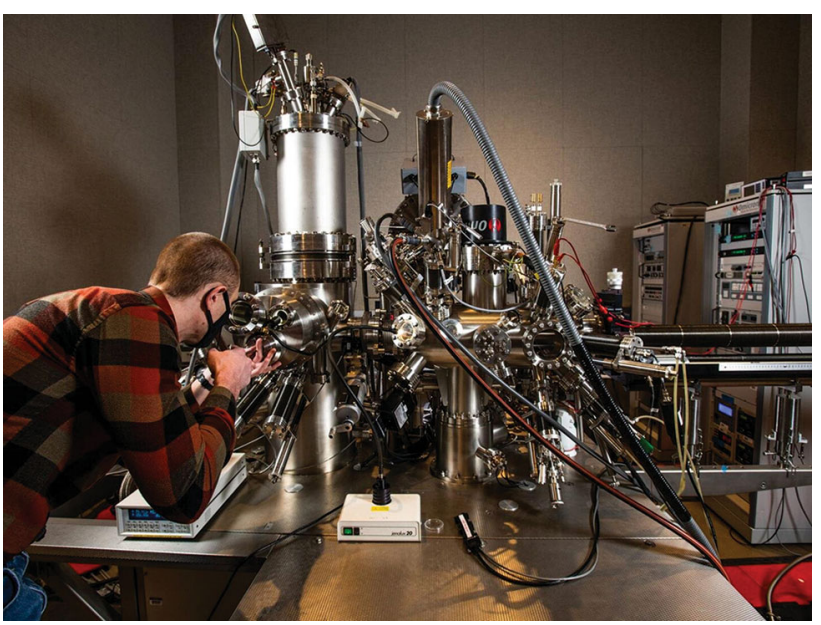

The ultra-high vacuum, low-temperature scanning probe microscope seen here will be moved to the new high-performance instrumentation lab in the Energy Sciences Center in 2022. Courtesy of Andrea Starr, Pacific Northwest National Laboratory

In recent years, scientists have discovered that certain arrangements of individual atoms supported on a surface make them special. These distinctive materials confer useful properties.
For instance, some single atom catalysts show promise for promoting conversion of biomass and waste carbon at room temperature and at significantly lower costs not possible today. These single atom catalysts are the subject of intense research to figure out how to make them operate on a large scale over a long period of time. And those investigations require studying them, taking atomic-scale "pictures" of them, and correlating their structure to their activity.

"We are searching for metal-oxide supports with a high density of sites that interact strongly with catalytically active metal atoms,' said Zdenek Dohnálek, a PNNL chemist who studies the detailed structure and function of catalysts. "These kinds of catalysts are highly sought after for converting biomass into fuels and commodity chemicals. We are looking at how they evolve, move, and transform on surfaces as a function of temperature and time."

Answering these basic scientific questions has only become possible within the last few years. It has required the convergence of precision imaging, new sampling, and processing techniques like those that led to Kovarik's recent microscopy inventions, and the emergence of mathematical methods that can effectively explain what the experimentalists are seeing.

For more information: https://www.pnnl.gov/.

\section{Engineers Develop the First Anti-COVID-19 Stainless Steel}

A team led by Professor Mingxin Huang of the University of Hong Kong (HKU), in collaboration with Professor Leo Lit Man Poon's research team, has made significant breakthroughs in producing the first anti-COVID-19 stainless steel that kills the severe acute respiratory syndrome coronavirus 2 (SARS-Cov-2) on its surface. The anti-COVID-19 stainless steel can also inactivate the H1N1 virus and E.coli on its surface. 


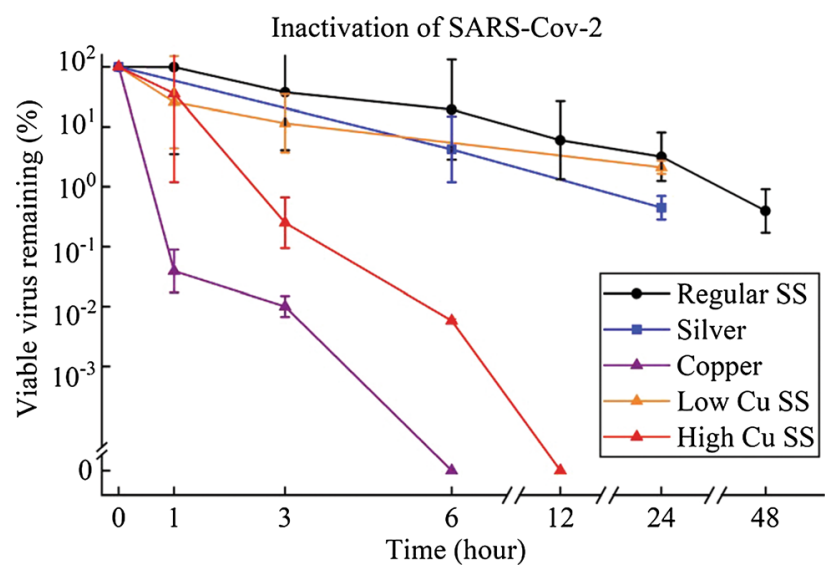

Viability of the SARS-Cov-2 on the surfaces of various metals (each point is the average value of three measurements). Credit: The University of Hong Kong

Stainless steel is one of the most extensively used materials in many public areas and hygiene facilities but has no inherent antimicrobial properties. Additionally, the SARS-CoV-2 exhibits strong stability on regular stainless-steel surfaces, with viable viruses detected even after three days. Undoubtedly, this has created a high possibility of virus transmission among people using these areas and facilities.

"In this latest breakthrough, the inactivation of pathogen microbes (especially the SARS-CoV-2) on a stainless-steel surface is achieved by tuning the chemical composition and microstructure of regular stainless steel,' Professor Mingxin Huang said.

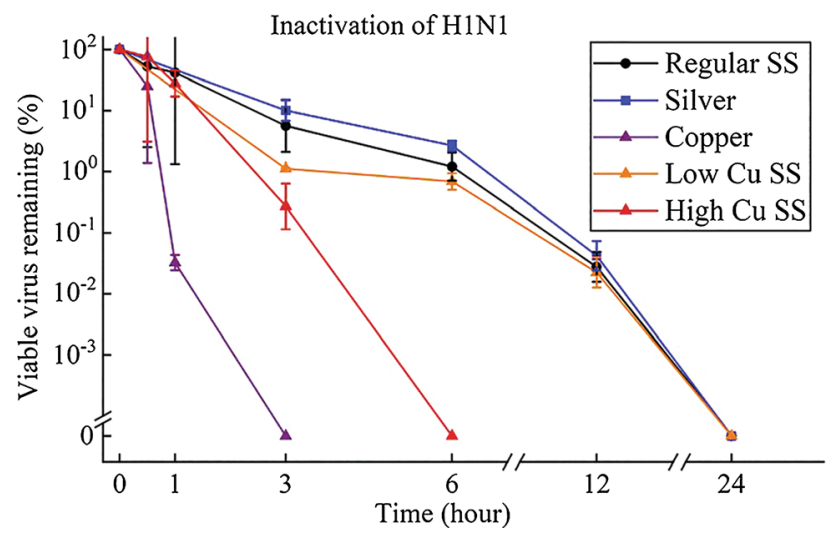

Viability of H1N1 on the surfaces of various metals (each point is the average value of three measurements). Credit: The University of Hong Kongx



(a)
Regular SS

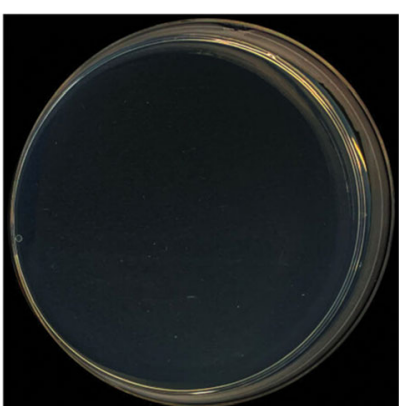

(b)
High Cu SS
Photos of typical bacterial colonies on (a) regular SS and (b) the high $\mathrm{Cu}$ SS (20 wt \%). Credit: The University of Hong Kong

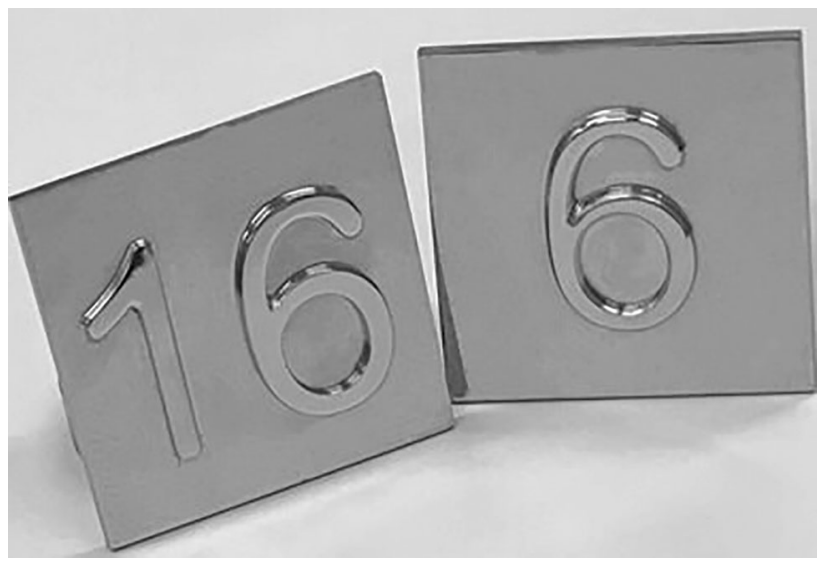

Lift buttons made from the high Cu SS (20 wt\%) by PM technology. Credit: The University of Hong Kong

A patent (Patent Cooperation Treaty (PCT) type) has been filed for the research findings. The team has been liaising with industrial partners to generate prototypes of public stainless-steel products such as lift buttons, doorknobs, and handrails for further tests and trials. For initial demonstration purposes, "floor buttons" used in lifts were fabricated successfully using high $\mathrm{Cu}$ stainless steel (20 wt\%) (Fig. 4).

For more information: https://www.sciencedirect.com/science/ article/pii/S1385894721053572?via\%3Dihub.

\section{Inspecting, Testing, and Measuring SiC}

Achieving the auto industry's stringent zero-defect goals is becoming a big challenge for makers of silicon carbide substrates, which are struggling to achieve sufficient yields and reliability as they migrate from 150 to $200 \mathrm{~mm}$ wafers and shift their focus away from pure silicon. 
$\mathrm{SiC}$ is a combination of silicon and harder carbide materials, and it has emerged as a key technology for battery electric vehicles due to its wide bandgap. Silicon carbide works at higher power, higher temperatures, and higher switching frequencies than silicon. Those properties can be leveraged to increase the range of EV batteries and shorten the charging time.
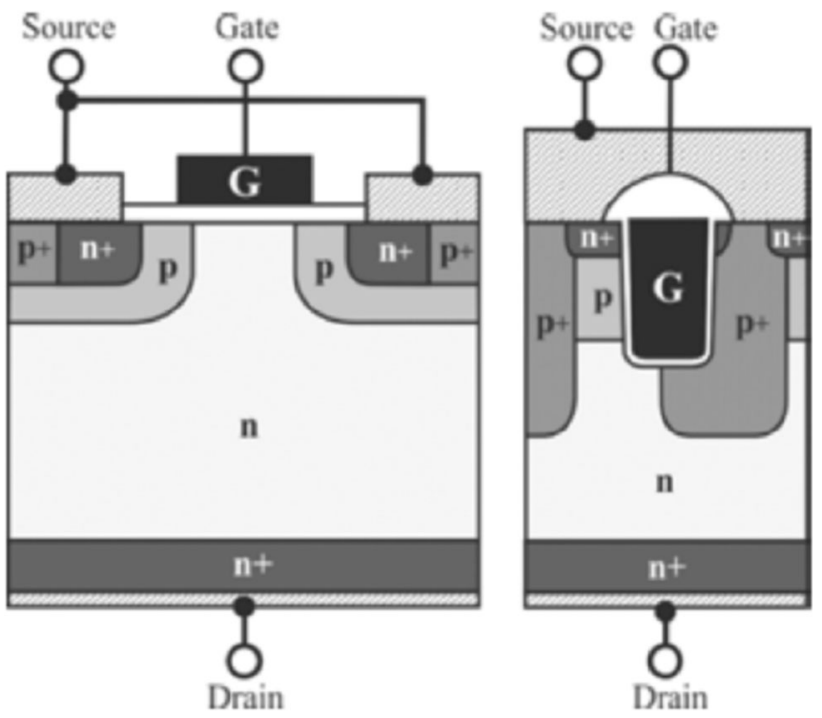

SiC device structures, planar-gate MOSFET (left) and trench MOSFET (right). Courtesy of Infineon

Silicon carbide is in high demand for a variety of applications, particularly in the automotive sector where it is ideal for electric vehicle power conversion chips due to the high breakdown field strength, thermal conductivity and efficiency.

Common testing today includes high magnification inline optical analysis to detect defects, statistical analysis of electrical parameters, and pattern analysis of wafer maps. In some cases, further physical analysis such as chemical delayering and focused ion beam analysis (FIB) may be required.

Other techniques like x-ray diffraction (XRD) also are being deployed for $\mathrm{SiC}$. XRD is used to characterize crystalline materials. Deployed in the industry for years, XRD first made inroads in the semiconductor industry for logic. It was, and still is, used to characterize silicon germanium materials in devices.

But the processes to identify and control defects still need some work.

Some of this is due to a shift to larger wafer sizes, and by way of comparison, the ramp from 200 to $300 \mathrm{~mm}$ for bulk silicon was difficult. This is compounded by the fact that $\mathrm{SiC}$ is being used increasingly in safety-critical applications, where defects can result in injury or death. Also in automotive applications, carmakers are demanding that chips function to spec for as long as 18 years and there is extreme pressure to reduce costs by improving yield.

For more information: https://semiengineering.com/ inspecting-testing-and-measuring-sic/.

\section{SECO/WARWICK to Deliver Furnace for Hardening Aviation Steel}

SECO/WARWICK, Meadville, Pennsylvania, will deliver a Vector vacuum furnace to one of the largest European machinery groups. The device will be used for hardening and tempering processes, and its design has been customized to meet the needs of a special and demanding process which is the hardening of aviation steel.

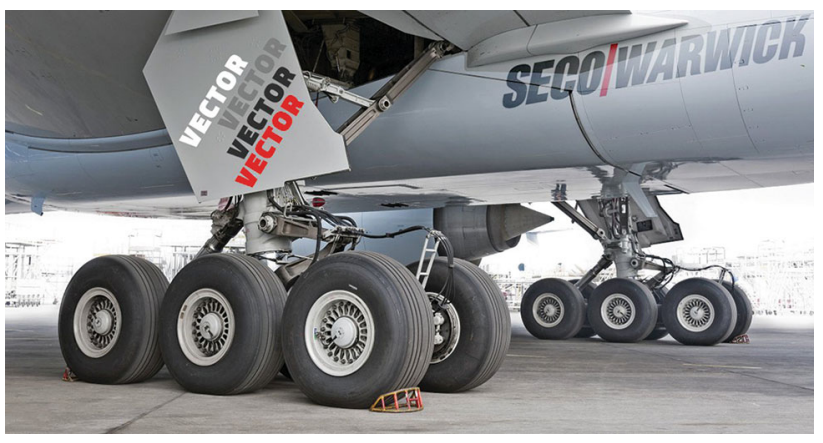

SECO/WARWICK's vector vacuum furnace, customized solution for aviation steel in the hardening of aircraft landing gear

This is the fourth SECO/WARWICK vacuum device for this manufacturer of heavy equipment. The Vector vacuum furnace on order is a proven design with the most popular dimensions for a working space, that is 600 by 600 by $900 \mathrm{~mm}$ ( 24 by 24 by 35 in.).

Vector vacuum furnaces can be used for the majority of standard hardening, tempering, annealing, solution heat treating, and brazing processes. However, this equipment can always be adapted to the specific requirements of the customer and thus to the particular industry. This furnace had to be adapted as far as possible to a dedicated thermal process used in the production of aircraft landing gear. Therefore, SECO/WARWICK engineers fitted the device with a nonstandard system for sub quenching with liquid nitrogen which enables the required quick cooling down of landing gear components. The solution on order has also been expanded with a vacuum system designed with a diffusion pump. This greatly increases the system performance. The furnace is equipped with a directional cooling option and convection heating system with a specially designed fan.

For more information: http://www.secovacusa.com/nrhardening-aviation-steel.

\section{Using Neutrons to Analyze Modified High-Entropy Metal Alloys}

Researchers at Oak Ridge National Laboratory have developed a method of adding nanostructures to high-entropy metal alloys, or 




ORNL researchers used neutrons at the lab's Spallation Neutron Source to analyze modified high-entropy metal alloys with enhanced strength and ductility, or the ability to stretch, under high-stress without failing. Credit: Rui Feng/ORNL, U.S. Dept. of Energy
HEAs, that enhance both strength and ductility, which is the ability to deform or stretch under tensile stress without failing.

The results, published in Science, open a promising pathway for tailoring HEA properties using small gradient structures to produce improved high-performance metals for a wide range of applications.

Conventional metallic materials, including HEAs (metals composed of five or more elements), become less ductile or more brittle as their strength increases.

The scientists used neutron diffraction methods at ORNL's Spallation Neutron Source to confirm tiny defect features, called stacking faults, form easily in an HEA compared to conventional fine-grained alloys.

"The stacking faults enhanced the test alloy's plasticity while also contributing to increased strength and hardening,"' said ORNL's Ke An. "Industries that could greatly benefit include automotive, power distribution and aerospace."

For more information: https://phys.org/news/2021-11neutrons-high-entropy-metal-alloys.html.

Publisher's Note Springer Nature remains neutral with regard to jurisdictional claims in published maps and institutional affiliations. 